\title{
Key challenges and opportunities faced by people with a primary immunodeficiency throughout the world
}

\author{
Jose Drabwell \\ Chair IPOPI, Firside, Main Road, Downderry, PL11 3LE, UK \\ E-mail: jose@ipopi.org
}

Changes take place all the time in the world of primary immunodeficiency. It really is an evolutionary path and in the developed western world the advances in technologies are looked upon as a near normal way of life. However in the areas of the world where there are so many challenges just to stay alive, the emphasis has to shift to the more pressing needs of the entire population. There are countries in Africa where there is not enough water. And if there is not enough water nor wood to start a fire how will it be possible to achieve even the most basic hygiene. Boiled water?

So diagnosis and treatment of people with a primary immunodeficiency are not going to get a priority in the severely restricted health services of those countries. Conflicts, AIDS and starvation are now part of life in some African and Asian countries. When an important advancement has taken place, such as the formation of the African Society for Immunodeficiencies (ASID), it can have a huge impact on the lives of people with a primary immunodeficiency. Suddenly immunologists, doctors, nurses, parents of children with a PID and patients themselves have a voice and a conduit to much information and assistance from the world wide PID community. Isolation, often associated with rare disorders, is no longer the issue.

There is a constant need to raise awareness and promote the urgency of diagnosis, but also to increase the visibility of this rare condition and to dispel the suspicion and so often the mistaken identification and stigma of HIV. Sadly this is not confined to underdeveloped countries, it still happens in London, Paris and many other sophisticated cities. Raising awareness amongst the first line of medical practitioners, such as GPs, lung specialists, ear, nose and throat specialists and gastroenterologists still has to be a matter of the utmost urgency. However dentists, ophthalmic surgeons, allergy and skin specialists should be made aware of these conditions as well, because blocked tearducts, skin lesions that will not heal, and receeding gums all could indicate a problem with an impaired immune system.

Once diagnosed there should follow a life saving programme of replacement immunoglobulin therapy. But what happens when such treatments are not readily available? In many countries there are inadequate diagnostic services available to obtain a diagnosis and that problem can be compounded when there is no health budget in place to buy the life saving therapies that could be available or to facilitate 
the provision of a national fractionating unit. The sobering fact is that more than $90 \%$ of people with a primary immunodeficiency are not diagnosed and hence are not receiving life saving therapy.

So as a global PID community we have to develop a strategy to help and support those countries where the need arises and which do not have the infrastructure in place so that immediate action can be taken. Challenging governments and working closely together with immunologists, patient organisations, existing relevant NGOs and affiliated experts to improve all aspects of primary immunodeficiencies has to be the way forward.

Naturally the priority should be focused on the person with a primary immunodeficiency. IPOPI - International Patient Organisation for Primary Immunodeficiencies is instigating the development of a "progressive treatment plan" to deal with those situations where no immediate replacement immunotherapy is available. A maintenance level of antibiotics, anti-fungal or anti-viral medication to be prescribed until immunoglobulin replacement therapy can begin. There should be a graduating treatment possibility from zero to gold standard.

The worldwide economic situation could exacerbate the problem with supply and reimbursement of expensive immunoglobulin. Most governments are involved in keeping their economy afloat and ultimately this will affect health budgets, so it is up to the global primary immunodeficiency community to ensure that sufficient statistics and data are collected so that a concerted and structured approach can be made to national governments to inform and advise health ministers of the need for a dedicated treatment plan for primary immunodeficiencies.

To show that with a successful regime of replacement immunoglobulin therapies a person can live a full and healthy life and consequently will gain employment, pay their taxes and as a result will help with the economy of the country. An employed primary immunodeficient patient has a better quality of life as well as a greater life expectancy and will therefore not be as great a burden on the state. The repeated and often expensive investigative procedures will become less, as well as the frequency of hospitalizations.

Not all governments have the same political commitment to dealing with such a rare condition, so it is the duty of all stakeholders in the primary immunodeficiency world to reiterate that there is no prevention, only treatment or containment of this disorder and that the treatment actually works! PIDs have to be looked upon as a public health issue and should receive the recognition of those healthcare providers in the national governments.

There are many brilliant scientists, immunologists and other medical personnel working on a variety of therapies to treat PIDs. Some, such as gene therapy, has already shown positive results, but with biotechnology, stem cell transplantation, genome advancement and innovative treatments being developed, it could lead to new and much more targeted treatment in the years to come. More clinical research into the various PIDs is so vital and obstacles which can delay and sometimes postpone this research must be removed. 
Obtaining ethical approval is vital, but the process could be simplified and should be speeded up to facilitate the development of orphan drugs in small populations like PIDs.

Primary immunodeficiencies might not have a voice in each country, but globally it is a different matter. Together with ESID, ASID, LASID, IUIS, INGID and all the patient organisations, along with PPTA and IPFA and their member companies, which provide the life saving immunoglobulins, IPOPI no longer has to whisper it can shout and will be heard.

Places far away from each other, such as India, Argentina, South Africa, Japan, Brazil etc are now joined together with Europe and the USA and exchange information and gain strength from the knowledge that IPOPI represents all national member organisations, regardless whether they are the well established members or just a fledgling start-up group.

The translation of the specific PID booklets into French, German, Spanish, Italian and Russian shows IPOPI's commitment to the international development of its organisation.

This, followed by the translation of IPOPI's e-newsletter (e-update) has made much information accessible to a large part of the world. Accessibility by the national member organisations to obtain information via the IPOPI website about the available immunoglobulin (intravenous and subcutaneous preparations) in their country, is of huge value, empowers the patient and reinforces the work of the clinicians.

In order to support those areas of the world where there is a need for national patients organisations, IPOPI has commenced a programme of regional meetings, which was started in 2008 with a very successful tour of South Africa, which will continue with a presence at the Eastern European J Project meetings, visits to North African countries and will culminate this year in Latin America in October 2009.

When the next bi-annual meeting takes places in Istanbul in October 2010, IPOPI hopes to have increased the number of national patient organisations by at least 10 more countries.

True to our vision IPOPI is working to improve the quality of life for people with primary immunodeficiencies and this will spread to even more corners of the world. 